\title{
Compact and Robust Fabry-Perot Cavity Antenna with PEC Wall
}

\author{
Jae-Gon Lee*
}

\begin{abstract}
In this paper, a novel Fabry-Perot cavity (FPC) antenna with a perfect electric conductor (PEC) wall is proposed to design a structurally compact and robust high-gain antenna. Generally, the FPC antenna comprising a PEC ground and a partially reflective dielectric surface (PRDS) is required to have a half-wavelength height to satisfy the resonance condition. If a perfect magnetic conductor (PMC) is substituted for the PEC ground, the height of the FPC antenna can be reduced to a quarter wavelength. The PRDS of the proposed FPC antenna is located on the PEC ground to obtain the effect of a PMC. Moreover, PEC walls are employed to block leakage by a guided mode inside the PRDS. As a result, the proposed FPC antenna can be designed as a compact high-gain antenna although it is composed of PEC ground and PRDS. To verify its feasibility, we simulated and measured the performance of the proposed antenna regarding the reflection coefficient, peak gain, and far-field radiation pattern. Finally, the height of the proposed antenna was reduced by approximately $50 \%$ compared with the conventional antenna, while the peak gain is more than equal to that of the conventional antenna.
\end{abstract}

Key Words: Fabry-Perot Cavity Antenna, High-Gain Antenna, Perfect Magnetic Conductor, Perfect Electric Conductor Wall.

\section{INTRODUCTION}

High-gain antennas are very useful and attractive in modern wireless technology such as $5 \mathrm{G}$ wireless communication, satellite communication, and microwave wireless power transfer. Various types of antennas can be used to achieve high-gain performance. Array antennas are the most popular solutions to overcome the low gain of an antenna. However, this type of antennas has some drawbacks such as complex feeding network with a loss that cannot be ignored and mutual coupling between array elements. In the case of a parabolic antenna, the required optimum focal length makes the antenna bulky. In 1956, the Fabry-Perot cavity (FPC) antenna was researched by Trentini [1] to achieve highgain performance. The FPC antenna achieves a maximum gain when the phases of transmitted powers by multiple reflections between a partially reflective surface (PRS) and a ground plane are in-phase. Since the FPC antenna has to satisfy the resonance condition, it has the minimum height of a half wavelength and is not inherently planar. Various types of low-profile FPC antennas have been introduced to overcome this weakness [2-10]. However, specific structures such as metasurfaces are needed to modify the resonance condition of the cavity; hence, the design procedure is complicated and difficult in these cases. Moreover, the gain of the conventional FPC antenna is proportional to the reflection magnitude and dimension of the PRS. When the reflection magnitude of the PRS increases, the dimension of the PRS and the ground plane should be simultaneously larger to obtain a maximum gain improvement $[11,12]$. Furthermore, the

Manuscript received October 16, 2020 ; Revised December 22, 2020 ; Accepted January 19, 2021. (ID No. 20201016-166J)

Department of Electronic Engineering, Kyungnam University, Changwon, Korea.

"Corresponding Author: Jae-Gon Lee (e-mail: jaegonlee@kyungnam.ac.kr)

This is an Open-Access article distributed under the terms of the Creative Commons Attribution Non-Commercial License (http://creativecommons.org/licenses/by-nc/4.0) which permits unrestricted non-commercial use, distribution, and reproduction in any medium, provided the original work is properly cited.

(c) Copyright The Korean Institute of Electromagnetic Engineering and Science. 
FPC antenna needs a supporter to hold the PRS above the ground.

A novel compact and robust FPC comprising a partially reflective dielectric surface (PRDS) and a perfect electric conductor (PEC) ground is proposed in this paper. Because the PRDS of the proposed FPC antenna is located on the PEC ground plane, the PRDS can be operated like a perfect magnetic conductor (PMC) from a radiating source. Thus, the height of the proposed FPC antenna can be decreased to a quarter-guided wavelength using only a PEC ground and a dielectric sheet. A guided mode inside the PRDS owing to a high permittivity is also generated and propagated to the side. Four PEC walls are employed to block the leakage, resulting in gain improvement. The design procedure of the proposed antenna is described in Section II. In Section III, the performances of the antenna, such as reflection coefficient, peak gain, and far-field radiation pattern, are simulated and measured to confirm our concepts.

\section{DESIGN OF COMPACT AND ROBUST FABRY-PEROT}

\section{CAVITY ANTENNA}

When a plane wave generated and propagated from region $I$ is incident normally at the interface between air and dielectric, as shown in Fig. 1(a), the reflection coefficient $(\Gamma)$ can be expressed as:

$$
\Gamma=\frac{\eta_{0} \sqrt{\frac{\mu_{2}}{\varepsilon_{2}}}-\eta_{0} \sqrt{\frac{\mu_{1}}{\varepsilon_{1}}}}{\eta_{0} \sqrt{\frac{\mu_{2}}{\varepsilon_{2}}}+\eta_{0} \sqrt{\frac{\mu_{1}}{\varepsilon_{1}}}}
$$

where $\varepsilon_{1}\left(\varepsilon_{2}\right)$ and $\mu_{1}\left(\mu_{2}\right)$ are the relative permittivity and permeability at region $\mathrm{I}(\mathrm{II})$, respectively. If the relative permittivities of regions I and II are 1 and infinity, respectively, the reflection coefficient is -1 . In other words, the amplitude and phase of the reflection coefficient are 1 and $-180^{\circ}$, respectively. Thus, when the plane wave is incident from air into the dielectric with a moderately high relative permittivity, the reflection property of the dielectric is nearly the same as that of a PEC. On the contrary, when the plane wave normally propagates from region II to I, as shown in Fig. 1(b), the reflection coefficient at the interface can be expressed as:

$$
\Gamma=\frac{\eta_{0} \sqrt{\frac{\mu_{1}}{\varepsilon_{1}}}-\eta_{0} \sqrt{\frac{\mu_{2}}{\varepsilon_{2}}}}{\eta_{0} \sqrt{\frac{\mu_{1}}{\varepsilon_{1}}}+\eta_{0} \sqrt{\frac{\mu_{2}}{\varepsilon_{2}}}}
$$

In this case, the reflection property at the interface between air and the dielectric is nearly the same as that of a PMC. The reason is that the amplitude and phase of the reflection coefficient are 1 and $0^{\circ}$, respectively, if the dielectric has a very high relative permittivity.

Fig. 2 shows a FPC by ground and PRS with excitation inside

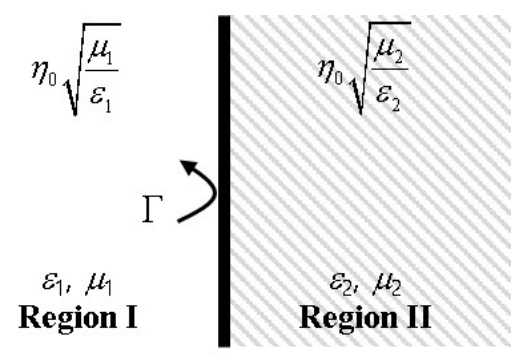

(a)

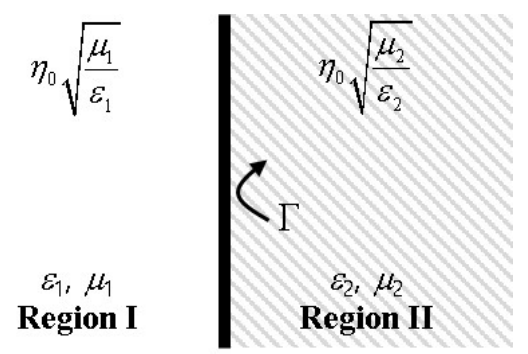

(b)

Fig. 1. Geometry of a plane wave normally incident at the interface between air and dielectric: (a) incidence from region I and (b) incidence from region II.

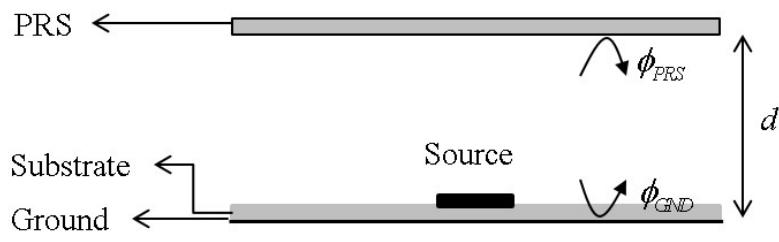

Fig. 2. Fabry-Perot cavity by ground and PRS with excitation inside a cavity.

a cavity. The directivity of the FPC antenna is given by [1]:

$$
D=\frac{1-\Gamma_{P R S}^{2}}{1+\Gamma_{P R S}^{2}-2 \Gamma_{P R S} \cos \left(\phi_{P R S}+\phi_{G N D}-\frac{4 \pi}{\lambda} d\right)} f^{2}
$$

where $\Phi_{P R S}$ and $\Phi_{G N D}$ are the reflection phases of the PRS and ground plane, respectively, $f$ is the radiation pattern of the source. From Eq. (3), $\phi_{P R S}+\phi_{G N D}-\frac{4 \pi}{\lambda} d$ and $\Gamma_{P R S}$ should be $2 \pi N(N=$ integer) and close to 1 , respectively, to achieve maximum directivity. Then, the height (d) of the FPC antenna is calculated by Eq. (4) and can be determined by the sum of the reflection phases of the ground plane and PRS.

$$
d=\left(\phi_{P R S}+\phi_{G N D}\right) \frac{\lambda}{4 \pi}+N \frac{\lambda}{2}
$$

For instance, if the PRS is implemented by a dielectric and the ground of the antenna is a PEC, the minimum height $(d)$ becomes half wavelength, as shown in Fig. 3(a). Moreover, when the $\mathrm{PEC}$ ground is changed into an artificial magnetic conductor (AMC), the height of the FPC antenna can be decreased to a 


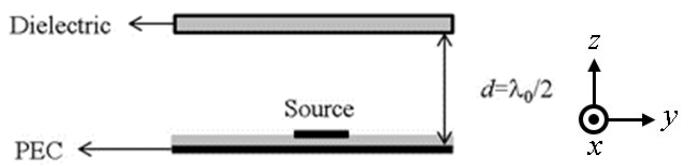

(a)

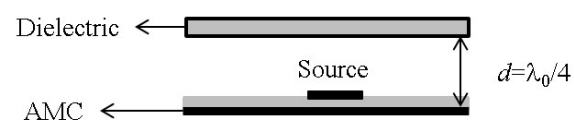

(b)

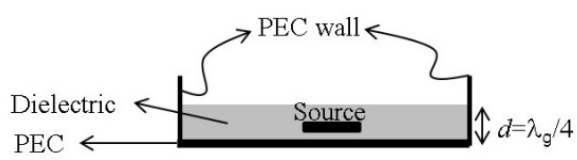

(c)

Fig. 3. Structure and height of the FPC antennas against various PRSs and ground planes: (a) dielectric above the PEC ground, (b) dielectric above the AMC ground, and (c) dielectric on the PEC ground (proposed antenna).

quarter wavelength because of the AMC reflection phase of $0^{\circ}$. In [3], the AMC ground can have a reflection phase from $180^{\circ}$ to $-180^{\circ}$ unlike a PEC ground, so that a low-profile FPC antenna, of which the height in this paper is $\lambda_{0} / 16$, can be designed by the combination of the AMC ground and PRS composed of a dielectric and patches. Similarly, a very low-profile FPC antenna having a height of $\lambda_{0} / 60$ can also be designed by the combination of the AMC ground plane and a metasurface having a reflection phase ranging from $180^{\circ}$ to $-180^{\circ}$ [4]. By controlling the sum of the reflection phases of the PRS and ground as in the previous research, the low-profile FPC antenna can be designed theoretically. However, it is difficult to design artificial surfaces such as $\mathrm{AMC}$ and metasurface.

Since the interface between air and the dielectric is operated as a PMC in the case of Fig. 1(b), the reflection phases of the ground $\left(\Phi_{G N D}\right)$ and $\operatorname{PRS}\left(\Phi_{P R S}\right)$ become $180^{\circ}$ and $0^{\circ}$, respectively. Therefore, the height of the FPC antenna is a quarter wavelength from Eq. (4). In this paper, a quarter-wavelength FPC antenna is proposed using a dielectric on the PEC ground, as shown in Fig. 3(c). However, a guided wave in this kind of structure propagates inside the dielectric slab so that the radiation from both edges of the dielectric slab is dominant as shown in Fig. 4(a) and the broadside gain is reduced. To achieve gain improvement, four PEC walls are employed in the proposed antenna, resulting in the reduction of end-fire radiation as shown in Fig. 4(b).

\section{Simulated AND MEASURED REsUlTS OF THE PRO- POSED FABRY-PEROT CAVITY ANTENNA}

The center frequency of the proposed antenna is $4.9 \mathrm{GHz}$, and the utilized substrate for the PRDS and source is RT/duroid

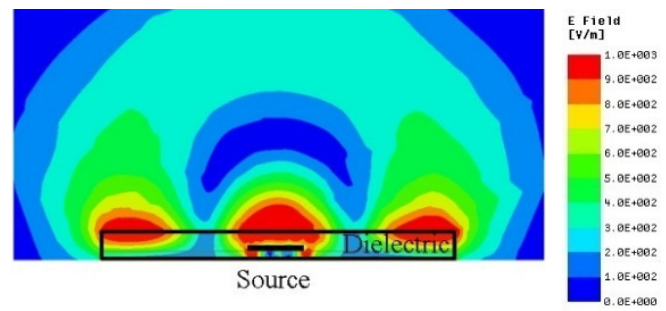

(a)

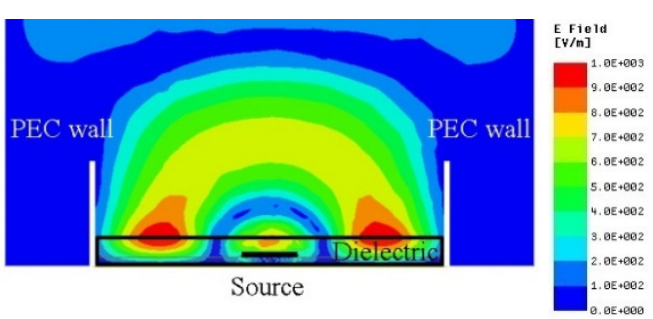

(b)

Fig. 4. E-field distribution of the proposed FPC antenna (a) without and (b) with PEC walls.

6010 with a relative permittivity of 10.2 . The thickness of the PRDS and substrate for the source are $4.8 \mathrm{~mm}$ and $1.6 \mathrm{~mm}$, respectively. Moreover, the designed source radiator for the proposed FPC antenna is a patch antenna with a length of $6.9 \mathrm{~mm}$ and width of $10 \mathrm{~mm}$. The feeding point of the coaxial cable is 2.6 $\mathrm{mm}$ away from the center, and the dimensions of the PRS and ground are $61.2 \mathrm{~mm}$ each in width and length, which corresponds with $1 \lambda_{0}$. The height of the conventional and proposed FPC antennas are $35.4 \mathrm{~mm}$ and $17.5 \mathrm{~mm}$ including a PEC wall, respectively. The peak gain of the proposed FPC antenna is proportional to the height of the PEC wall. However, when the height of the PEC wall is more than $17.5 \mathrm{~mm}$, the peak gain is saturated so that the height of the PEC wall becomes $17.5 \mathrm{~mm}$. A conventional patch antenna was employed as the source of the FPC antenna and was fed by a coaxial cable. Furthermore, the conventional $\lambda_{0} / 2$ and proposed $\lambda_{g} / 4$ FPC antennas were fabricated to compare their performances, as shown in Fig. 5. Fig. 6

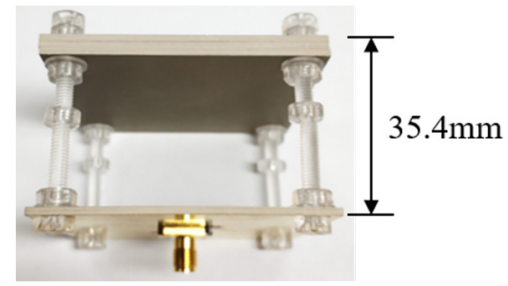

(a)

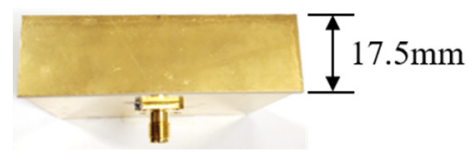

(b)

Fig. 5. Photographs of (a) the conventional and (b) proposed FPC antennas. 


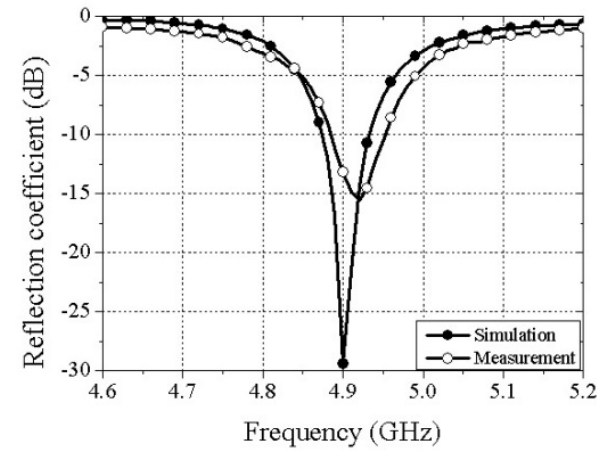

(a)

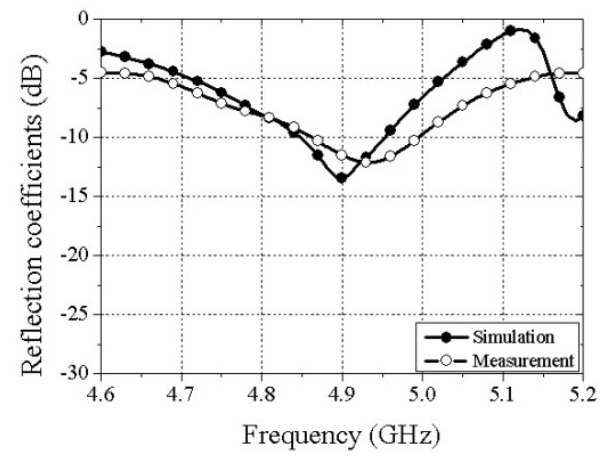

(b)

Fig. 6. Simulated and measured reflection coefficients of (a) the conventional and (b) proposed FPC antennas.

presents the simulated and measured reflection coefficients of the conventional and proposed FPC antennas. The measured results were obtained using Agilent $8510 \mathrm{C}$ vector network analyzer. The simulated and measured reflection coefficients of the proposed FPC antenna were $-13.5 \mathrm{~dB}$ and $-12.1 \mathrm{~dB}$ at $4.9 \mathrm{GHz}$ and 4.93 $\mathrm{GHz}$, respectively, which are relatively different from those of the conventional FPC antenna. To design a PRDS with a thickness of $4.8 \mathrm{~mm}$, three commercial substrates were stacked in the case of the proposed antenna. Therefore, it seems that the discrepancies between the simulated and measured results are caused by a manufacturing tolerance. The impedance matching of the proposed FPC antenna at the resonant frequency was poor compared to that of the conventional FPC antenna because the input impedance is modified by the dielectric of the PRDS [13]. In addition, the far-field radiation patterns and peak gains were measured in the anechoic chamber system to confirm the performance. The anechoic chamber is composed of a shield enclosure (size: 4 $\mathrm{m} \times 2.5 \mathrm{~m} \times 2.5 \mathrm{~m}$ ), 18 inch pyramidal absorber, network analyzer, wireless communication test set, positioner, turn table, and dual-polarized transmit antenna. The simulated and measured far-field radiation patterns of the conventional and proposed FPC antennas are compared in Fig. 7. Although the height of the proposed FPC antenna is nearly half compared with that of the conventional FPC antenna, the peak gain of the proposed FPC an-

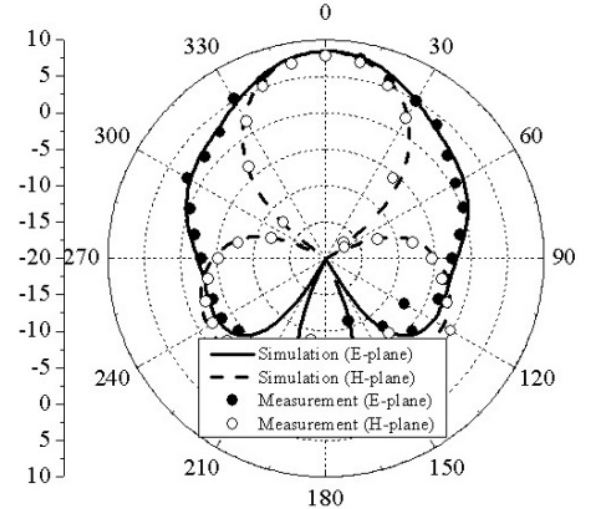

(a)

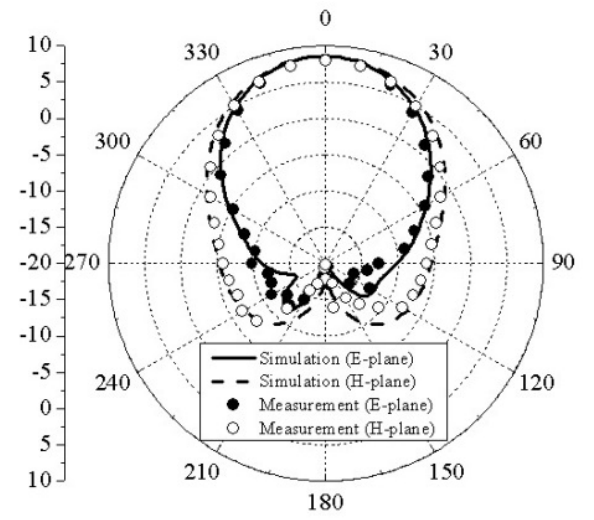

(b)

Fig. 7. Simulated and measured far-field radiation patterns of (a) the conventional and (b) proposed FPC antennas.

tenna was measured to be $7.98 \mathrm{dBi}$ and is almost similar to that of the conventional FPC antenna. The difference between the simulated and measured results seems to be caused by a coaxial cable loss and a misalignment between the source radiator and the PRDS. Moreover, Fig. 8 shows a comparison of the peak gains of the conventional and proposed FPC antennas against frequency. It was observed that the peak gain of the proposed antenna is broader than that of the conventional FPC antenna.

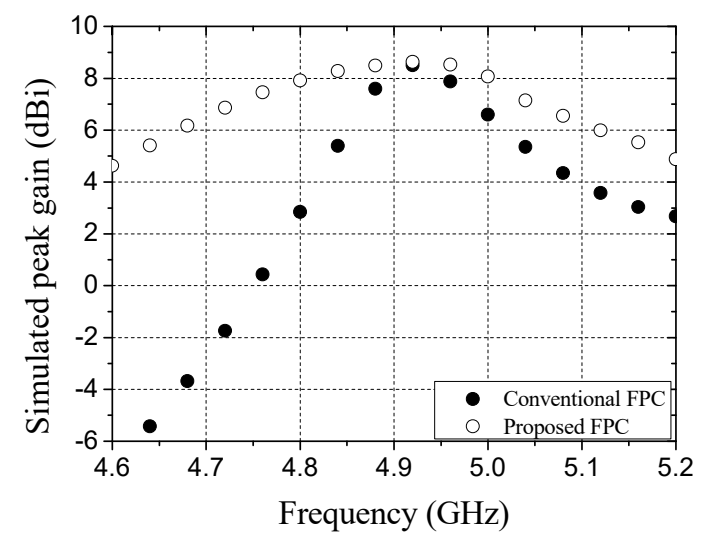

Fig. 8. Comparison between the peak-gains of the conventional and the proposed FPC antenna against frequencies. 


\section{CONCLUSION}

In this paper, a new type of low-profile FPC antenna was proposed using a PRDS with a PMC effect. The proposed antenna is structurally robust because the PRDS is directly located on the PEC ground and PEC walls are integrated to prevent leakage. The prototype is designed and fabricated using a substrate with a relative permittivity of 10.2 at $4.9 \mathrm{GHz}$. Even if the height of the proposed FPC antenna is nearly half compared with that of the conventional FPC antenna, the peak gain of the proposed FPC antenna is more than equal compared with that of the conventional FPC antenna. From the measured results, it is concluded that the proposed FPC antenna can be one of the solutions for a compact and robust high-gain antenna.

This work was supported by Kyungnam University Foundation Grant, 2019.

\section{REFERENCES}

[1] G. V. Trentini, "Partially reflecting sheet arrays," IRE Transactions on Antennas and Propagation, vol. 4, no. 4, pp. 666-671, 1956.

[2] A. P. Feresidis, G. Goussetis, S. Wang, and J. C. Vardaxoglou, "Artificial magnetic conductor surfaces and their application to low-profile high-gain planar antennas," IEEE Transactions on Antennas and Propagation, vol. 53, no. 1, pp. 209-215, 2005.

[3] L. Zhou, H. Li, Y. Qin, Z. Wei, and C. T. Chan, "Directive emissions from subwavelength metamaterial-based cavities," Applied Physics Letters, vol. 86, article no. 101101, 2005. https:// doi.org/10.1063/1.1881797

[4] H. Li, J. Hao, L. Zhou, Z. Wei, L. Gong, H. Chen, and C. T. Chan, "All-dimensional subwavelength cavities made with metamaterials," Applied Physics Letters, vol. 89, article no. 104 101, 2006. https://doi.org/10.1063/1.2338795

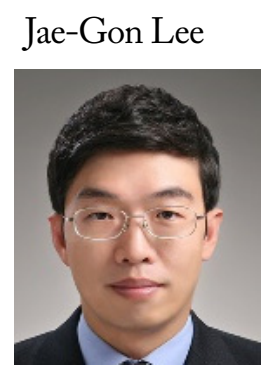

received his B.S., M.S., and Ph. D. degrees in Electronic and Electrical engineering from Hongik University, Seoul, Korea, in 1999, 2001, and 2007, respectively. From 2007 to 2015 , he was a chief researcher at LG Electronics, and from 2016 to 2018, he was a research professor at Metamaterial Electronic Device Research Center, Hongik University. In 2019, he joined the Department of Electronic Engineering, Kyungnam University, Changwon, Korea, where he is currently an assistant professor. His research interests include metasurface/ metamaterial RF devices, small antennas, and EM sensors.
[5] J. Ju, D. Kim, W. Lee, and J. Choi, "Low-profile Fabry-Perot cavity antenna with a double-layered partially reflecting surface structure," Microwave and Optical Technology Letters, vol. 53, no. 2, pp. 271-273, 2011.

[6] Z. Z. Yang, F. Liang, Y. Yi, D. Zhao, and B. Z. Wang, "Metasurface-based wideband, low-profile, and high-gain antenna," IET Microwaves, Antennas \& Propagation, vol. 13, no. 4, pp. 436-441, 2019.

[7] J. G. Lee and J. H. Lee, "Low-profile Fabry-Perot cavity (FPC) antenna using meta-surface for dual-band," in Proceedings of 2017 International Symposium on Antennas and Propagation (ISAP), Phuket, Thailand, 2017, pp. 1-2.

[8] Z. Liu, S. Liu, B. Bian, X. Kong, and H. Zhang, "Metasurfacebased low-profile high-gain substrate-integrated Fabry-Perot cavity antenna," International Journal of $R F$ and Microwave Computer-Aided Engineering, vol. 29, no. 4, article no. e21583, 2019. https://doi.org/10.1002/mmce.21583

[9] F. Deng and J. Qi, "Shrinking profile of Fabry-Perot cavity antennas with stratified metasurfaces: accurate equivalent circuit design and broadband high-gain performance," IEEE Antennas and Wireless Propagation Letters, vol. 19, no. 1, pp. 208-212,2020.

[10] Y. Liu, Y. Hao, and S. Gong, "Low-profile high-gain slot antenna with Fabry-Perot cavity and mushroom-like electromagnetic band gap structures," Electronics Letters, vol. 51, no. 4, pp. 305-306, 2015.

[11] J. G. Lee, T. S. Kwon, and J. H. Lee, "Effects of finite superstrate and asymmetrical ground on high gain superstrate antenna," IEICE Transactions on Communications, vol. 101B, no. 8, pp. 1884-1890, 2018.

[12] T. S. Kwon, J. G. Lee, and J. H. Lee, "The gain estimation of a Fabry-Perot cavity (FPC) antenna with a finite dimension," Journal of Electromagnetic Engineering and Science, vol. 17, no. 4, pp. 241-243, 2017.

[13] A. Bhattacharyya, "Effects of dielectric superstrate on patch antennas," Electronics Letters, vol. 24, no. 6, pp. 356-358, 1988. 